
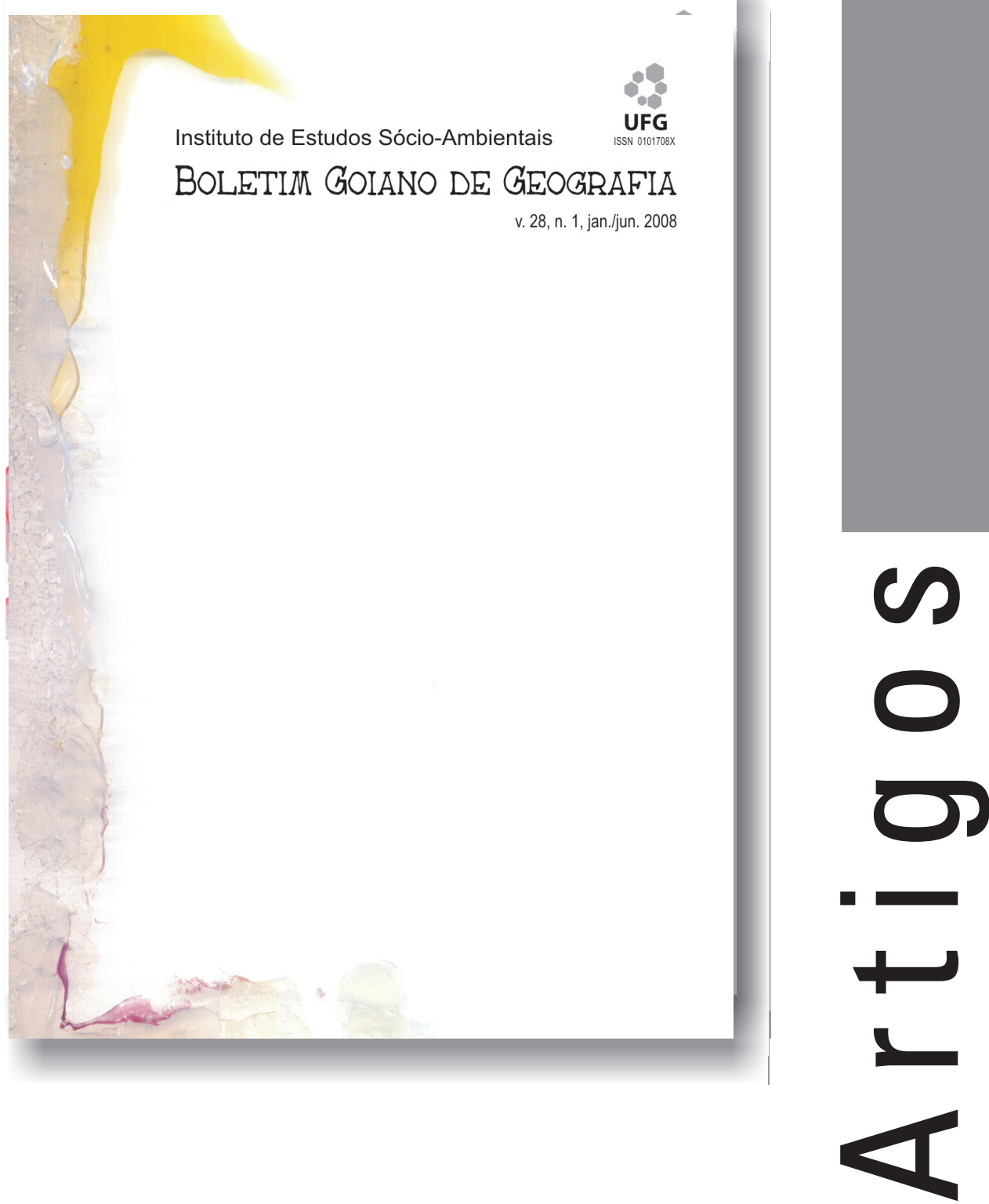


\title{
A influência de elementos macro estruturais no processo de meteorização em solos saprólitos
}

\author{
The influence of macrosturural features in the metereorization process in saprolith soils
}

Bruno M. Martins - Universidade de Coimbra

geogbruno@gmail.com

Resumo

0 papel de elementos macro estruturais, como a presença de blocos de rocha sã embutidos em perfis de solos saprólitos, a presença de litóclases, elementos de fraqueza como fileiras de minerais de argila ou contactos geológicos contribuem para uma heterogeneidade que caracteriza este tipo de formações superficiais. 0 seu estudo reveste-se de enorme importância na evolução meteórica e na dificuldade em definir o nível de estabilidade das vertentes regularizadas por este tipo de formações superficiais que, por sua vez, são as mais vulneráveis à ocorrência de movimentos em massa.

Vários estudos apontam para que a avaliação do grau de estabilidade dos solos saprólitos tenha por base a análise do solo como um todo, evitando uma análise isolada do fabric do perfil.

Neste artigo, procuramos descrever as principais modificações macroscópicas, químico-mineralógicas e granulométricas em dois perfis talhados em rochas granitóides, cujo papel de elementos macro estruturais é decisivo na evolução meteórica.

Palavras-chave: processo de metereorização, solo saprólitos

\begin{abstract}
The role of macrostructural features such as large particles, lithoclasys, secondary clay seems or geological contacts often contribute to heterogeneities in various forms and scales of saprolitic soils. This large variability in type, abundance and scales of heterogeneities in saprolitic profiles means that critical features control stability of slopes.
\end{abstract}

Several studies indicate that macrostructural features in saprolitic soils are in many cases of greater importance for the behaviour of the soil mass and that the profile characteristics must be considered as a whole.

In this article, we will try to describe the most significant macroscopic, chemical, mineralogical and granulometric modifications in two igneous saprolites profiles whose macrostructural features undergo a weakening process during weathering.

Key-word: metereorization process, saprolith soils

\begin{tabular}{|l|l|l|l|l|l|l|}
\hline Boletim Goiano de Geografia & Goiânia - Goiás - Brasil & v. 28 & n. 1 & p. $29-42$ & jan. / jun. & 2008 \\
\hline
\end{tabular}




\section{Introdução}

O termo saprólito é entendido neste artigo, no seu sentido mais lato, como que designando todo o material resultante da metereorização in situ, e não apenas o horizonte superficial mais evoluído, "saprolith" (Aydin et al, 2000). A análise do fabric de um solo saprólito é importante uma vez que revela as condições que estiveram na sua génese, condicionada por aspectos morfoclimáticos passados e actuais, assim como as características da rocha sã.

Os solos saprólitos são caracterizados por inúmeras variações mineralógicas, assim como a presença de litóclases, intrusões, blocos de rocha sã embutidos, zonas de contacto litológico, contribuindo para uma elevada heterogeneidade.

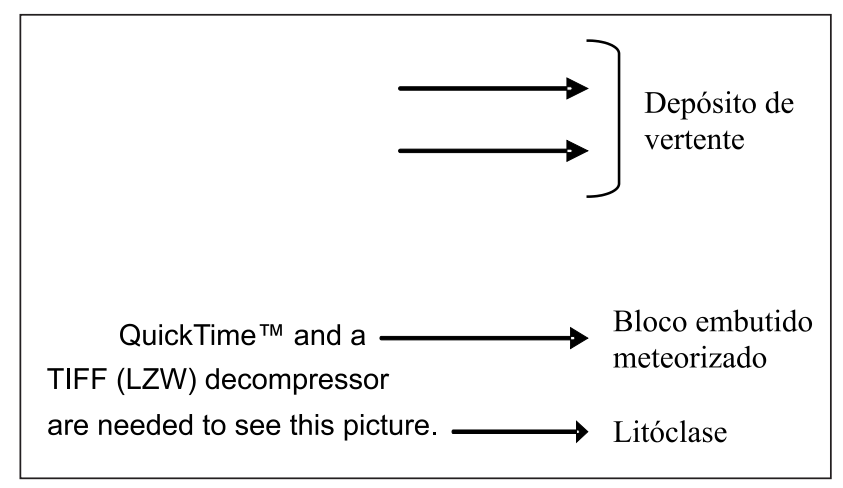

Figura 1 - Perfil de um solo saprólito

Vários estudos tem demonstrado dificuldade na avaliação da estabilidade de vertentes regularizadas por solos saprólitos e ao mesmo tempo, a importância destes no desenvolvimento de movimentos em massa.

Estudos recentes revelam ainda a importância da análise do solo como um todo na avaliação do seu comportamento e não apenas a análise específica do fabric, reforçando a acção dos aspectos macro estruturais (Costa et al, 1989), nomeadamente o papel das litóclases e descontinuidades geológicas associadas a movimentos em massa (John et al, 1969; Vargas \& Pichler, 1957; Massad \& Teixeira, 1985; Costa \& Fernandes, 1985; Cowland \& Carbray, 1988; Irfan \& Woods, 1988; Chigira, 2001; Wen \& Aydin, 2003). O papel das litóclases, diáclases, falhas e fracturas, principalmente de orientação sub-vertical em solos saprólitos de rochas ígneas, é evidenciado em inú- 
meros estudos como factor muito importante na dinâmica hidro-geológica, permitindo uma maior infiltração em profundidade e consequentemente, anomalias piezométricas que surgem imediatamente após a queda de precipitação (Aydin, 2006).

Neste artigo, analisamos as principais modificações macroscópicas, físico-químicas e mineralógicas a partir da análise de dois perfis localizados em rochas granitóides cujo papel de litóclases e da tectónica reforça o carácter complexo intrínseco deste tipo de formações superficiais.

\section{Localização e geologia da área de estudo}

Os perfis analisados localizam-se ao Norte de Portugal, na região de Vila Pouca de Aguiar e Chaves, caracterizada pelo predomínio de rochas granitóides, dominada pelo Plutão de Vila Pouca, que corresponde a um lacólito assente discordantemente em relaçãos às estruturas sin-D3 e aos metassedimentos do Silúrico e pelos granitos sin tectónicos D3.
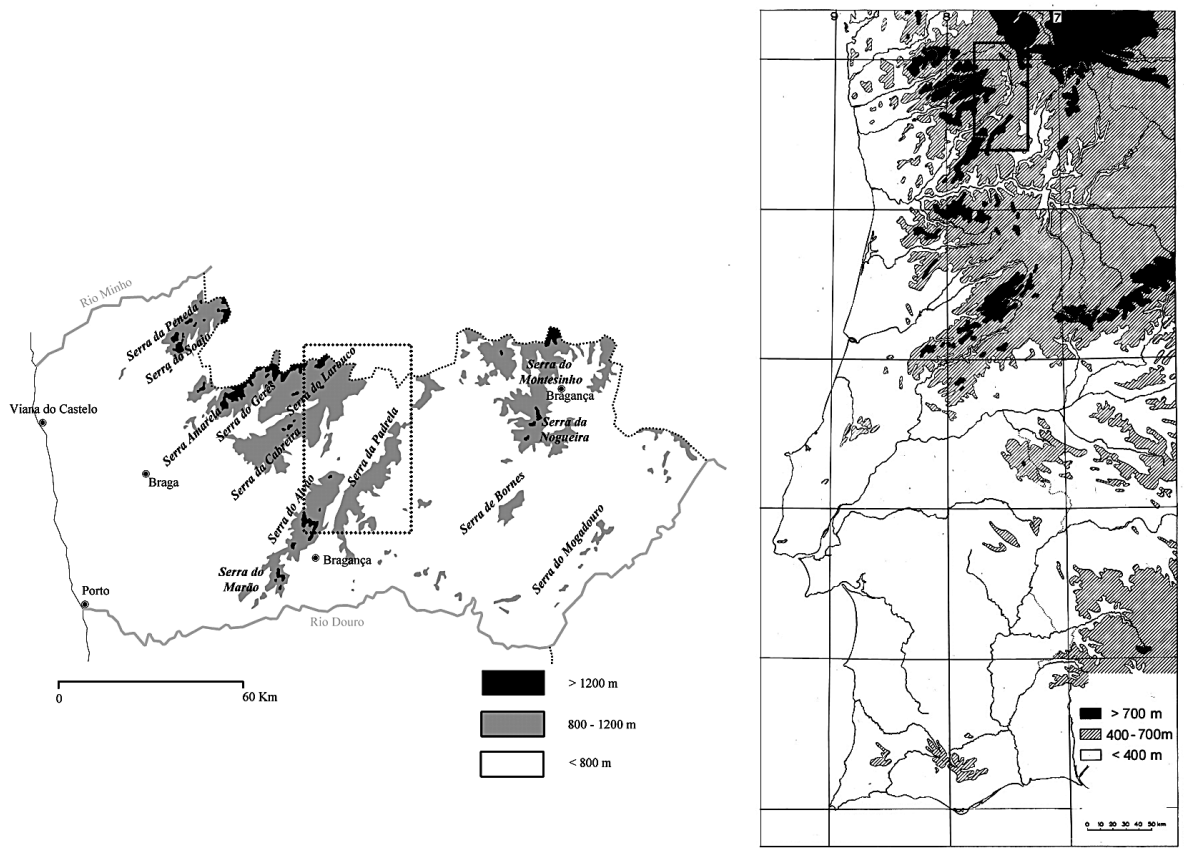

Figura 2 - Localização da área de estudo 
O Plutão de Vila Pouca apresenta uma forma alongada de orientação NNE-SSW, coincidente com a falha desligante Chaves-Verin, constituído por dois tipos de granito o granito de Vila Pouca e o de Pedras Salgadas. O granito de Vila Pouca de Aguiar apresenta geometria laminar (soleira) e foliações magnéticas inclinadas para o exterior do Plutão. Trata-se de um granito porfiróide de grão médio caracterizado pela presença de enclaves microgranulares tonalíticos e granodioríticos acompanhados frequentemente por filões de orientação concordante com o Plutão. O granito de Pedras Salgadas corresponde à ultima pulsação magnética e preenche a maior parte do Plutão. É mais leucocrata e menos biotítico que o granito de Vila Pouca. Apresenta mega cristais de feldspato potássico ou de tendência porfiróide de grão médio.

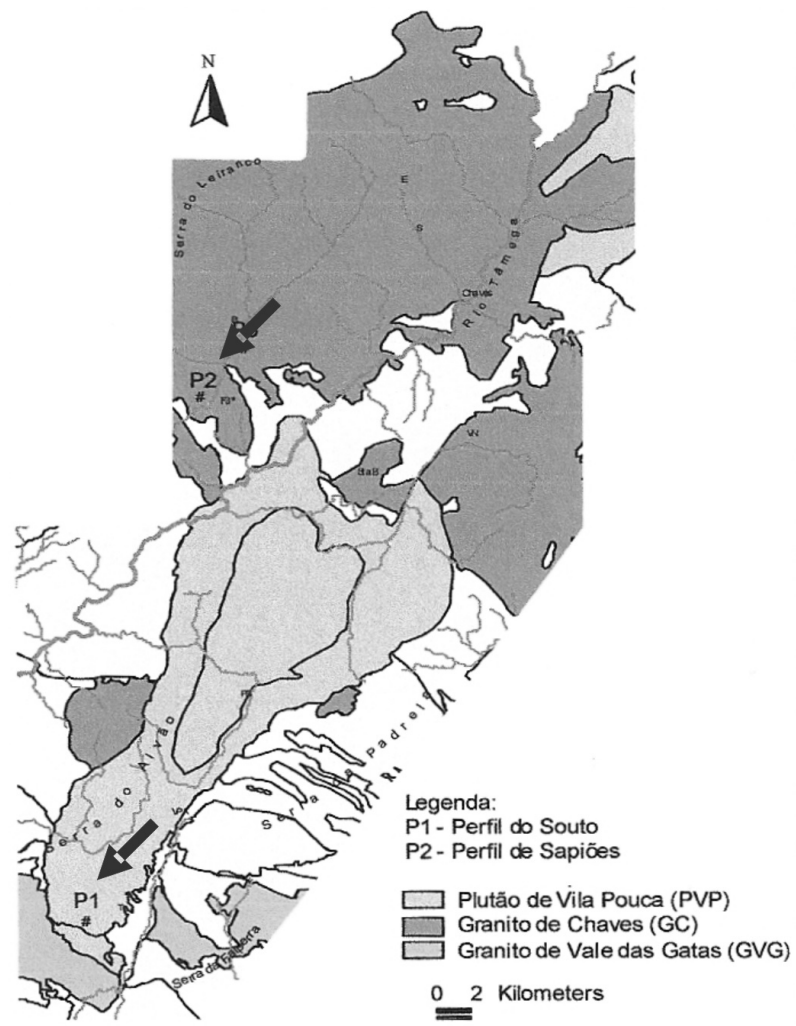

Figura 3 Localização dos perfis estudados 
O cálculo de alterabilidade diferencial em função da composição mineralógica $\mathrm{M}$ revela valores que variam entre os 85 e os 78 para o granito de Vila Pouca de Aguiar e entre os 72 e os 68 para o granito de Pedras Salgadas. Ainda com base nos elementos mineralógicos, da relação P/Fk obteve-se valores entre os 2,1 e os 1,8 para o granito de Vila Pouca de Aguiar e um valor médio de 1,6 para o granito de Pedras Salgadas. Ao nível dos aspectos químicos, o índice potencial de alteração WPI é ligeiramente superior no granito de Vila Pouca de Aguiar (11) do que para o granito de Pedras Salgadas (10).

O granito de Chaves ocupa principalmente a parte norte da área de estudo. Trata-se de um granito alcalino, de grão médio a grosseiro de duas micas essencialmente moscovítico. Contém plagioclase francamente albítica, fortemente meteorizada, caulinizado com pelo menos duas gerações de moscovite. A relação $\mathrm{P} / \mathrm{Fk}$ é de 0,9 o que indica valores de feldspato potássico relativamente altos face aos valores da plagioclase. Ainda tendo por base as características mineralógicas do granito, o valor obtido pelo índice de alterabilidade $\mathrm{M}$ é de 51,7. Do ponto de vista químico o granito de Chaves destaca-se pela percentagem elevada de sílica, ligeiramente superior a 70\%. O valor de RSA, que resulta da relação $\mathrm{SiO} 2$ / Al2O3, apresenta valores médios na ordem dos 4,7. Este valor é muito idêntico aos granitos que constituem o Plutão de Vila Pouca (PVP), ainda que ligeiramente mais baixo, relacionado principalmente aos os valores mais altos de AlO.

Análise de alguns perfis

O perfil do Souto (P1) localiza-se na vertente oriental da serra do Alvão a cerca de 600 metros de altitude e com cerca de 3 metros de altura visíveis. Não apresenta traços de meteorização esferoidal, tipificada pela presença de bolas graníticas. Uma litóclase de orientação NNE-SSW sub-vertical tornando-se progressivamente sub-horizontal, define dois níveis de meteorização diferenciados um próximo à litóclase, e outro afastado, funcionando como goteira, permitindo uma diferenciação meteórica no perfil.

No sector marginal à litóclase o granito é W4, caracterizado pela presença ainda de rocha sã. No geral o granito apresenta sinais de meteorização. A clivagem da rocha é geralmente feita pelas fileiras de biotite. As micas, principalmente a biotite, perderam o brilho, apresentando-se geralmente baças e com anéis de oxidação. Os feldspatos esboroam-se com alguma dificuldade e os minerais de quartzo conservam melhor orientação. O granito mais afastado da litóclase é W3, a rocha apresenta-se compacta, bastante resistente, partindo-se com dificuldade com o martelo.

Os minerais de quartzo e a moscovite surgem conservados. Os feldspatos não se esboroam, embora as biotites tenham perdido algum brilho. 
Tratando-se de estados de meteorização entre rocha fresca e granito muito decomposto, as séries granulométricas reflectem a predominância das areias grosseiras, correspondendo a um saibro típico de um fabric pouco evoluído. Surge amiúde a inclusão de rochas não desagregadas nas amostras recolhidas. A percentagem da fracção silto-argilosa é muito reduzida, resultante, principalmente, da alteração dos feldspatos e das plagioclases.

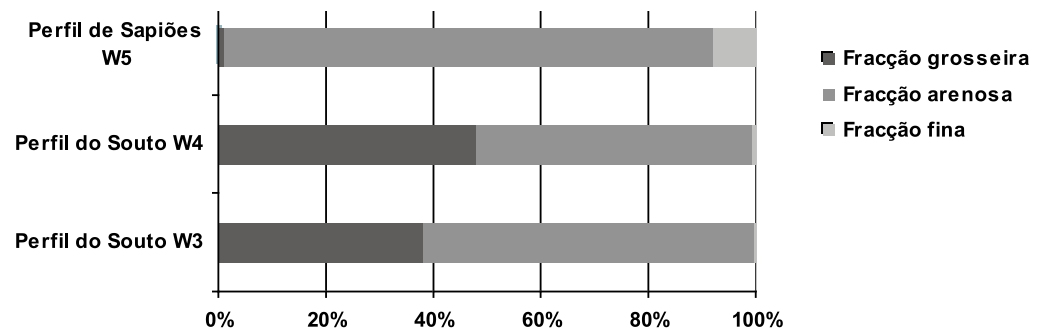

Figura 4 - Análise granulométrica dos perfis analisados

As séries granulométricas indicam valor de $3,1 \mathrm{~mm}$ para o valor da mediana no granito W4 e 3,8mm para o sector W3. Para o primeiro quartil o valor obtido nas amostras recolhidas no granito W4 é de $2,07 \mathrm{~mm}$ e $1,71 \mathrm{~mm}$ para o terceiro quartil. No granito W3 os resultados obtidos para o primeiro e terceiro quartil são $2,61 \mathrm{~mm}$ e $1,76 \mathrm{~mm}$ respectivamente.

Entre o granito W3 e W4, verifica-se do ponto de vista químico, um aumento de sílica, uma diminuição geral dos óxidos, principalmente do $\mathrm{Na}_{2} \mathrm{O}$. Observa-se ainda um ligeiro aumento do $\mathrm{K}_{2} \mathrm{O}$ e a acumulação do teor de alumínio, relacionado com o facto deste óxido ficar provavelmente retido, em grande parte, nos produtos de metereorização (Figura 5)

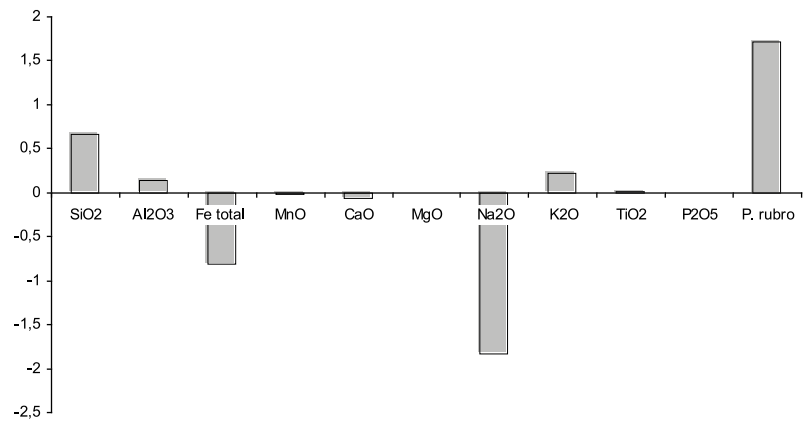

Figura 5 - Variação química entre o granito marginal e próximo à diaclase 
A litóclase terá favorecido a lixiviação, evidenciada no cálculo do índice de lixiviação $\beta$. O índice varia entre 0 e 1, respectivamente para a rocha completamente alterada e para a rocha sã.

Foi calculado o índice de lixiviação para as amostras colhidas em granito W3 e W4, e posteriormente, comparadas com a rocha sã (figura 6):

(i) $\beta=\mathrm{ba}_{1} \mathrm{~W} 3 / \mathrm{ba}_{1}$ da rocha sã $\mathrm{e}=\mathrm{ba}_{1} \mathrm{~W} 4 / \mathrm{ba}_{1}$ da rocha sã

(ii) $\beta=\mathrm{ba}_{1} \mathrm{~W} 4 / \mathrm{ba}_{1} \mathrm{~W} 3$

em que

$\mathrm{ba}_{1}=\mathrm{K}_{2} \mathrm{O}+\mathrm{Na}_{2} \mathrm{O} / \mathrm{Al}_{2} \mathrm{O}_{3}$

Os resultados revelam um aumento do índice no sector próximo à litóclase $(0,69)$ em comparação com os valores obtidos no sector mais afastado $(0,89)$.
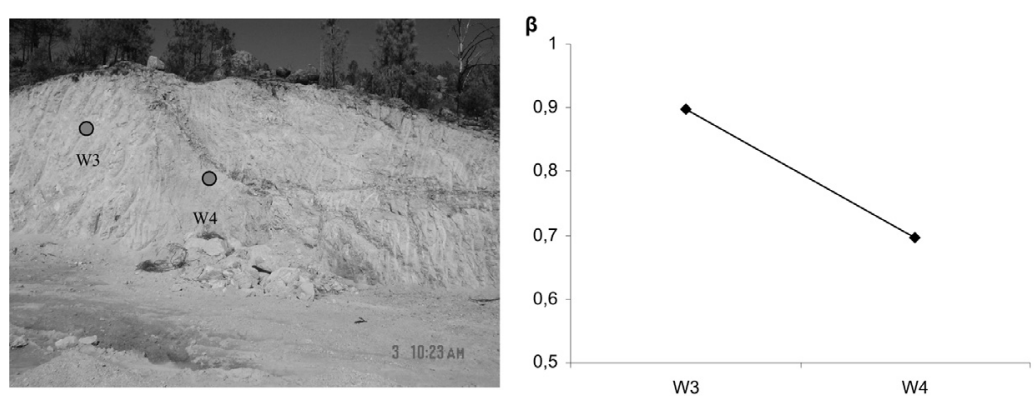

Estampa 1 - Localização da recolhas das amostras;

Figura 6 - Cálculo do índice de lixiviação $\beta$ para os granitos W3 (sector mais afastado da litóclase) e granito W4 (sector do granito mais próximo da litóclase)

Ao nível da fracção argilosa verificam-se ganhos de montmorilonite, na ordem dos $33 \%$, passando de $27 \%$ para $60 \%$. A arenização caracteriza-se assim pelo desenvolvimento deste mineral que, constituído por duas camadas de sílica, resulta da remoção dos catiões básicos e da retenção de parte da sílica como resíduo da metereorização (figura 8). A caulite apresenta valores semelhantes nas duas amostras, cerca de $25 \%$ da fracção lítica.

Ao norte, foram recolhidas amostras de granito alterado num perfil denominado por perfil dos Sapiões (P2), talhado em granito de Chaves. As amostras foram recolhidas numa zona tectonizada por falhas de orientação 
NNE-SSW acompanhadas de intrusões pegmatíticas e comparadas com a rocha sã no sentido de avaliar a intensidade de meteorização.

O granito no sector marginal mostra-se bem conservado, de grão médio a grosseiro, de duas micas, essencialmente moscovítico. Na zona de alteração, o granito surge completamente alterado (W5). O fabric apresenta uma textura porosa, os materiais micáceos, especialmente a biotite, apresentam esfoliação. E os materiais mais resistentes, o quartzo e a moscovite, surgem com evidentes sinais de deterioração. Os feldspatos esboroam-se facilmente com os dedos. A preservação da orientação mineralógica inicial é dada apenas pelo quartzo.

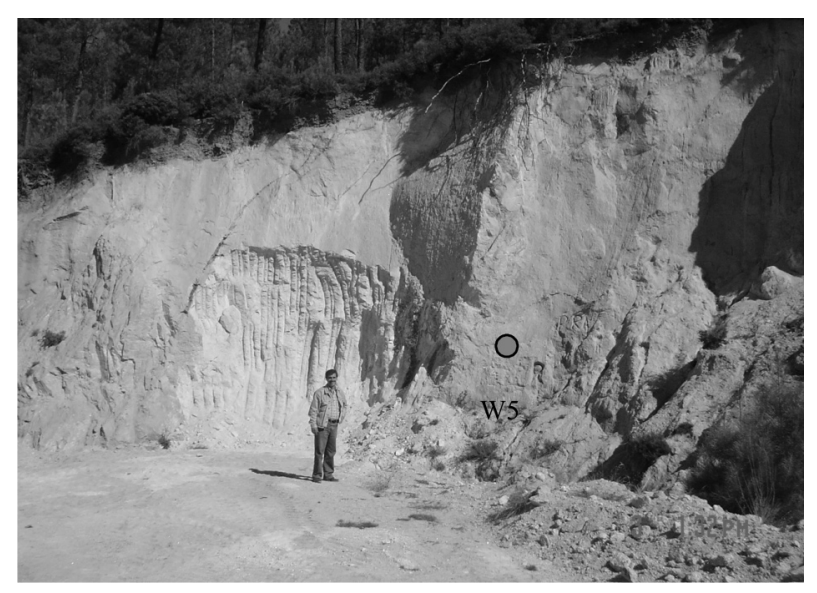

Estampa 2 - Perfil de Sapiões. É possível observar no interior da caixa de falha, intrusões pegmatíticas de orientação concordante à falha. Localização da recolha das amostras

O perfil corresponde a uma massa homogênea, preenchido por algumas intrusões pegmatíticas, não sendo possível encontrar bolas graníticas. As amostras colhidas para a elaboração do tratamento granulométrico foram obtidas a cerca de 4 metros de profundidade. Caracterizam-se por uma boa percentagem da fracção silto-argilosa e por uma boa calibragem granulométrica (figura 4).

Do ponto de vista químico, verifica-se um aumento da alumina, facto que estará relacionado com a sua retenção nos produtos de meteorização. Verificam-se perdas de sílica, indicando uma fase mais adiantada de meteorização, posterior à lixiviação dos elementos alcalinos e alcalino-terrosos (figura 7). 


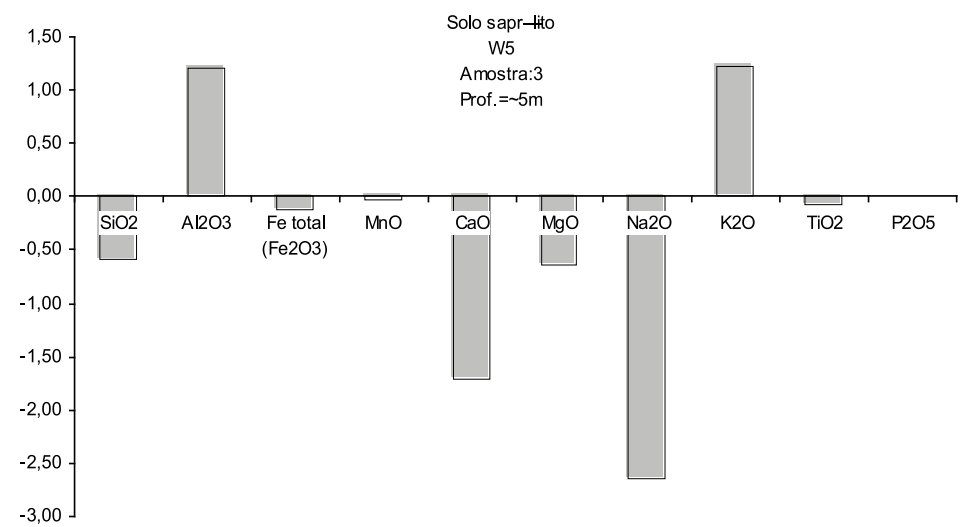

Figura 7 - Variação química entre o granito W5 e a rocha sã

Tabela 1 - Resultados obtidos de algumas relações moleculares

\begin{tabular}{|c|c|c|c|c|c|c|c|c|c|c|c|c|c|}
\hline & ba1 & ba & ba2 & ba3 & WPI & PI & WI & a & b & bi & ki & kr & sf \\
\hline W5 & 0,43 & 0,43 & 0,04 & 0,45 & 8,38 & 0,83 & 0,87 & 6,18 & 8,54 & 67,58 & 4,34 & 3,88 & 37,07 \\
\hline
\end{tabular}

Ao nível da fracção argilosa, a caulinite é o mineral mais representativo, cerca de $80 \%$ da fracção total. Esta percentagem reflecte o avanço meteórico do granito, sob condições de boa drenagem. Na tabela estão representadas ainda algumas relações moleculares como forma de avaliação do grau de metereorização.

\section{Discussão e conclusão dos resultados}

O papel de descontinuidades geológicas contribui de forma especialmente importante para o avanço da meteorização, facilitando o avanço do processo em profundidade, principalmente tratando-se de falhas ou diáclases de orientação vertical ou sub-vertical. Por outro lado, a sua presença é por si só elemento de fraqueza e de perda de coesão do solo.

O avanço da meteorização é acompanhado por uma diminuição progressiva da dimensão dos grãos, relacionado com a conversão de alguns minerais, como a plagioclase e a biotite, numa fracção silto-argilosa. Desta forma, ainda que isolada, a análise da granulometria do fabric é um indicador do estado de meteorização, principalmente da fracção fina. Convém, no 
entanto, reter que este de tipo de análise deve ter em consideração a textura inicial da rocha.

Do ponto de vista macroscópico, as principais alterações estão relacionadas com a descoloração e o brilho dos minerais, e a diminuição da coesão da estrutura inicial da rocha que vai progressivamente convertendo-se num solo, acompanhada de uma variação do volume da rocha.

Do ponto de vista mineralógico, o avanço da metereorização reflete-se inicialmente nos minerais mais susceptíveis, nomeadamente as plagioclases e a biotíte, contribuindo para a perda gradual da coesão da rocha. A biotite tem tendência a perder o brilho inicial, tornando-se gradualmente mais baça, perdendo flexibilidade e dureza. Os minerais de quartzo, mais resistentes, reflectem o avanço meteórico com o surgimento de uma pequena película marginal de sílica amorfa, ao passo que o feldspato potássico tem tendência a fragmentar-se como sinal deste avanço.

O avanço da metereorização reflecte-se ainda nas características da fracção argilosa do fabric, que evolui progressivamente de um processo de bissialitização para monossialitização.

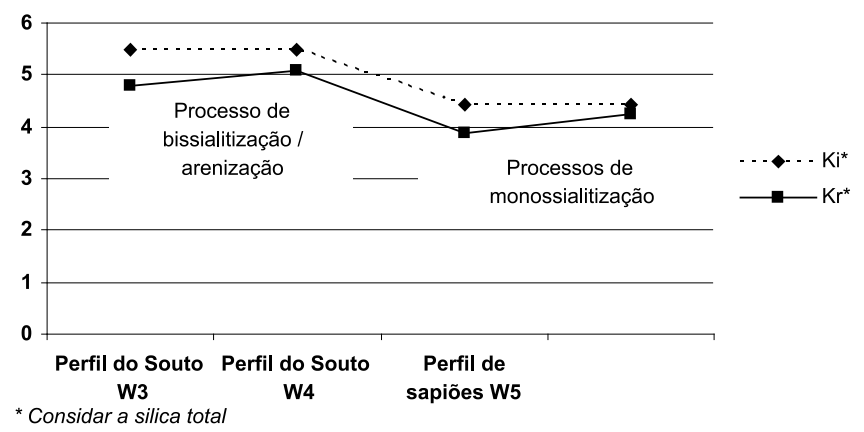

Figura 8 - Evolução da meteorização com base na análise das relações moleculares Ki e Kr para os perfis analisados

No entanto, as conclusões baseadas na análise mineralógica da fracção argilosa de um perfil terão que ser feitas com algumas reservas, dada a coexistência de minerais primários, sensíveis à metereorização, com minerais secundários evoluídos. Terá que ter em conta a paragênese de alguns minerais, e não apenas a presença e/ou ausência de determinados minerais. A percentagem da fracção argilosa é muito importante para dissecar este problema, assim como a quantidade dos minerais primários e o estado de conservação dos mesmos. Existe uma grande diferença entre a percentagem 
da fracção argilosa em perfis de alteração de zonas temperadas em comparação com perfis de zonas tropicais.

Também a análise química do fabric reflecte a metereorização de um maciço rochoso. $\mathrm{O}$ avanço meteórico envolve numa primeira fase a lixiviação de elementos alcalino e alcalino-terrosos $\left(\mathrm{K}_{2} \mathrm{O}, \mathrm{CaO}, \mathrm{Na}_{2} \mathrm{O}, \mathrm{MgO}, \mathrm{MnO}\right.$ e $\mathrm{FeO})$, e posteriormente, a lixiviação gradual de silício $\left(\mathrm{SiO}_{2}\right)$ e a acumulação de sesquióxidos $\left(\mathrm{Fe}_{2} \mathrm{O}_{3}, \mathrm{Al}_{2} \mathrm{O}_{3}, \mathrm{TiO}_{2}\right)$.

\section{Referências}

Aydin, A., Duzgoren-Aydin, N. S., and Malpas, J. A review of igneous and metamorphic saprolites, Journal of Nepal Geological Society, 22, 11-16. 2000.

Aydin, A. Stability of saprolotic slopes: nature and the role of field scale heterogeneities, Natural Hazards and Earth System Sciences, European Geosciences Union. 2006.

Chigira, M. Mirco-sheeting of granite and its relationship with landsliding specifically after the heavy rainstorm in June 1999, Hiroshima Prefecture, Japan, Engineering, Geology, 59, 2001.

Costa, A. J. and Fernandes, C. E. M. Slides in saprolites from gneissic rocks with relict slip surfaces, the B.N.E.D.E.S. case history, Proc. 1st Int. Conf. on Tropical Lateritic and Saprolitic Soils, 2, 1985.

Costa, L. M., Dobereiner, L., de Campos, T. M. P., and Vargas, E. Fabric and engineering properties of saprolites and laterites, Proc. 12th Int. Conf. SMFE, 4, 1989.

Cowland, J. W. and Carbray, A. M. Three cut slope failures on relict discontinuities in saprolitic soils, Proc. 2nd Int. Conf. on Geomechanics in Tropical Soils, 1, 1988.

Irfan, T. Y. and Woods, N. W. The influence of relict discontinuities on slope stability in saprolitic soils, Proc. 2nd Int. Conf. on Geomechanics in Tropical Soils, 1, 1988.

Massad, F. and Teixeira, H. R. Deep cut on saprolitic soils conditioned by relict structures, Proc. 1st Int. Conf. on Tropical Lateritic and Saprolitic Soils, 2, 1985.

St. John, B. J., Sowers, G. F., and Weavers, C. H. E. Slickensides in residual soils and their engineering significance, Proc. 7th Int. Conf. on SMFE, II, 1969.

Vargas, M. and Pichler, E. Residual soil and rockslides in Santos (Brazil), Proc. 4th Pan-American Conf on SMFE, 6/27, 1957.

Wen, B. P. and Aydin, A. Microstructural study of a natural slip zone, quantification and deformation history, Engineering Geology, 68, 2003.

Bruno M. Martins - Geógrafo da Faculdade de Letras da Universidade de Coimbra 